\title{
SURVEY ON POLYGONAL APPROXIMATION TECHNIQUES FOR DIGITAL PLANAR CURVES
}

\author{
Kalaivani Selvakumar and Bimal Kumar Ray \\ School of Information Technology and Engineering, \\ VIT University, Vellore, India. \\ kalaivanisevit.ac.in \\ bimalkumarray@vit.ac.in
}

\begin{abstract}
Polygon approximation plays a vital role in abquitious applications like multimedia, geographic and object recognition. An extensive number of polygonal approximation techniques for digital planar curves have been proposed over the last decade, but there are no survey papers on recently proposed techniques. Polygon is a collection of edges and vertices. Objects are represented using edges and vertices or contour points (ie. polygon). Polygonal approximation is representing the object with less number of dominant points (less number of edges and vertices). Polygon approximation results in less computational speed and memory usage. This paper deals with comparative study on polygonal approximation techniques for digital planar curves with respect to their computation and efficiency.
\end{abstract}

\section{KEYWORDS}

Polygon approximation, contour points, dominant points, digital planar curves.

\section{INTRODUCTION}

In recent decades, the applications of image processing and computer vision are heuristically increased. For various types of application like object recognition, industrial dimension inspection and many other engineering and science fields. Extracting consequential information from the digital planar curves in many machine vision applications is the important target. Consequential information means where the point has high curvature and high responsibility for representing the shape of that object. These points are called as dominant points. The dominant points are used to represent the shape with less number of contours and retain the shape.

Famous Attneave's [4] observation is that Consequential information about shape is concentrated at dominant points of a curve. Those techniques provide a essential data reduction while preserving important information about object which are used for image matching, pattern recognition and shape description [5].

Shape representation by polygonal approximation consists of serious vertices. These representations become very popular for its simplicity, locality, generality and compactness [6]. Polygonal approximation is used in various applications like, 
- The representation of numerals on car number plates and aircraft by Goyal et al[7].

- Electrooculographic (EOG) biosignal processing by semyonov [8]

- Shape understanding[4]

- By set of feature points achieve image analysis by [9]

- Image matching algorithm.[10,11,12]

The goal of polygonal approximation is to capture the boundary shape with least number of vertices. In this approach, some techniques achieve optimal polygon approximation but with maximum error value. $[13,14,15,16]$.

In most of the techniques, the user defined the threshold examine the number and location of the vertices in the polygon. Some other techniques obtain the vertices directly using dominant point detection and approximate the polygon. Generally, dominant points are points with high curvature.

In section 2 the techniques are explained, in section 3 the results are compared with measures.

\section{COMPARATIVE STUDY}

\subsection{Technique 1}

Asif Masood[1].This approach approximates the polygon in iterative manner. By using the freeman's chaincode, it starts extract the breakpoint from the given contour points.

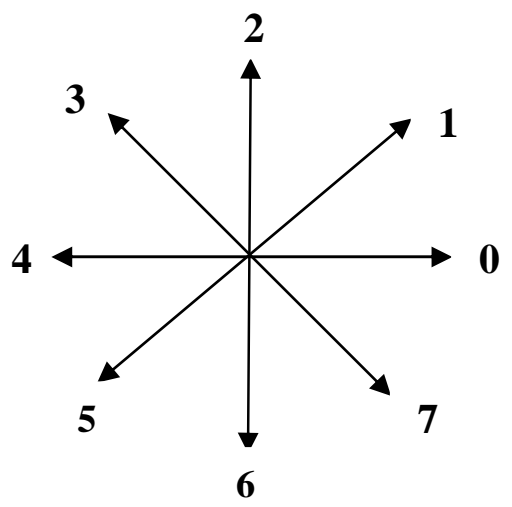

Fig1:Freeman's chaincode 


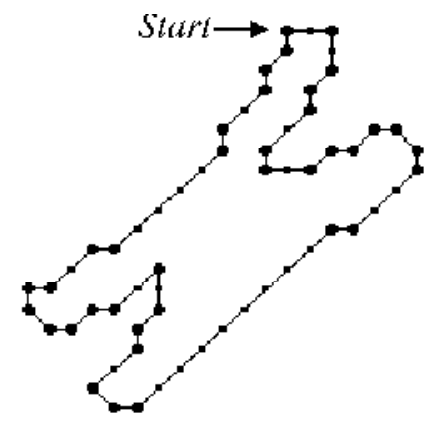

Fig 2: After Freeman's chaincode chromosome breakpoints (in bold)

The figure 1[ref12] shows the freeman's chaincode shows all possible direction of the points. Consider figure 2, the chromosomes mark with start indicates the start direction for freeman's chaincode.

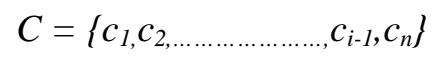

$P=\left\{\left(x_{1}, y_{1}\right),\left(x_{2}, y_{2}\right), \ldots \ldots\left(x_{i-1}, y_{i-1}\right),\left(x_{n}, y_{n}\right)\right\}$

$i=\{1,2,3, \ldots \ldots \ldots \ldots \ldots i-1, n\}$

where $\mathrm{C}$ is a collection of freeman's chaincode value (from 0 to 7 ) and $n$ represents the number of contour points. The $\mathrm{P}$ is a collection of contour point's $x$ and $y$ co-ordinates for $n$ number of contour points.

Table 1. Freeman's Chaincode

$\begin{array}{|lr|}\text { If } C_{i} & \neq C_{i+1} \text { Then } \\ & P_{i} \text { is a breakpoint. } \\ \text { Else } & \\ \text { End } & P_{i} \text { is not a breakpoint. }\end{array}$

The associated error value (AEV) is calculated using those extracted breakpoints. The perpendicular (squared) distance between point $P_{k}$ to the straight line $P_{i}$ and $P_{j}$ is called associated error value (AEV) .Take sequence of points as $\mathrm{P}_{\mathrm{i}}, \mathrm{P}_{\mathrm{j}}$ and $\mathrm{P}_{\mathrm{k}}$ and calculate the AEV as follows,

Table 2.Calculation of AEV

1. Join $P_{i}$ and $P_{j}$ as a straight line

2. Draw perpendicular line from $P_{k}$ point which joins the line $P_{i}$ and $P_{j}$

3. Calculate perpendicular squared distance where

$P_{i} \equiv\left(x_{i}, y_{i}, P_{j} \equiv\left(x_{j}, y_{j}\right)\right.$ and $P_{k} \equiv\left(x_{k}, y_{k}\right)$ 


$$
\frac{\left(\left(x_{k}-x_{i}\right)\left(y_{j}-y_{i}\right)-\left(y_{k}-y_{i}\right)\left(x_{j}-x_{i}\right)\right)^{2}}{\left(x_{i}-x_{j}\right)^{2}+\left(y_{i}-y_{j}\right)^{2}}
$$

The AEV is calculated for each break point and stored. The algorithm compares the AEV sequence and identified the least AEV's break point to be deleted. This iteration process deletes all redundant breakpoint. This technique produces expected quality result but still suboptimal, to address this stabilization process is introduced. Stabilization algorithm relocates the break points by comparing its integrated square error (ISE). Integrated square error (ISE) is calculated as follows:

$$
I S E=\sum_{i=1}^{n} e_{i}
$$

where $e_{i}$ is the squared distance of $i$ th point $P$ of the approximated polygon. This process compares ISE values of its predecessor and successor and relocates the break point where its shape doesn't make more change. It reduces the total distortion (error) which is caused by its neighboring vertices. By this stabilization process the dominant point (DP) moves to optimal location and produces optimal approximated polygon. This approximation technique results at any compression ratio or required error or at any finite approximated measurement.

Table 3: Masood's polygonal approximation algorithm through stabilization process

Number of contour points is $N$
Apply chaincode using Table 1
Calculate AEV for all Contours using Table 2
While(termination condition) and store
Drop DP with least AEV
Mark flag for neighboring DPs
Apply stabilization procedure
do
List $=$ collection of unstable DPs
If empty (List)
Return ()
End if
For each unstable DP
SP=set stable position
If SP $\neq$ current position of DP
Move DP to SP


Mark flag for neighboring DPs

End if

Get unmark DP

End for

While (no unstable points)

List $=$ get repositioned DPs

Recalculate and update the AEV for List

This approach iteratively deletes the redundant point and stabilization process, stabilizes the points in right location with lass error value and produce high quality polygon approximation.

\subsection{Technique 2}

Carmona [2] proposed a new breakpoint suppression algorithm which suppresses the redundant point and detects the dominant point effectively. This approach first applies freeman's chaincode and extracted initial breakpoints using [ref2]. Then perpendicular distance is calculated using [refence].Select any breakpoint from the set of breakpoints which is extracted using chaincode. Assume a threshold value $d_{t}$ which is less than one. Now compare the perpendicular distance $d$ sequence with threshold value $d_{t}$ if this $d$ value is less than $\mathrm{d}_{\mathrm{t}}$, remain that points which is liable to that distance. This iteration follows until the final point is equal to initial points.

Table 4: Carmano's polygonal approximation algorithm through break point suppression process

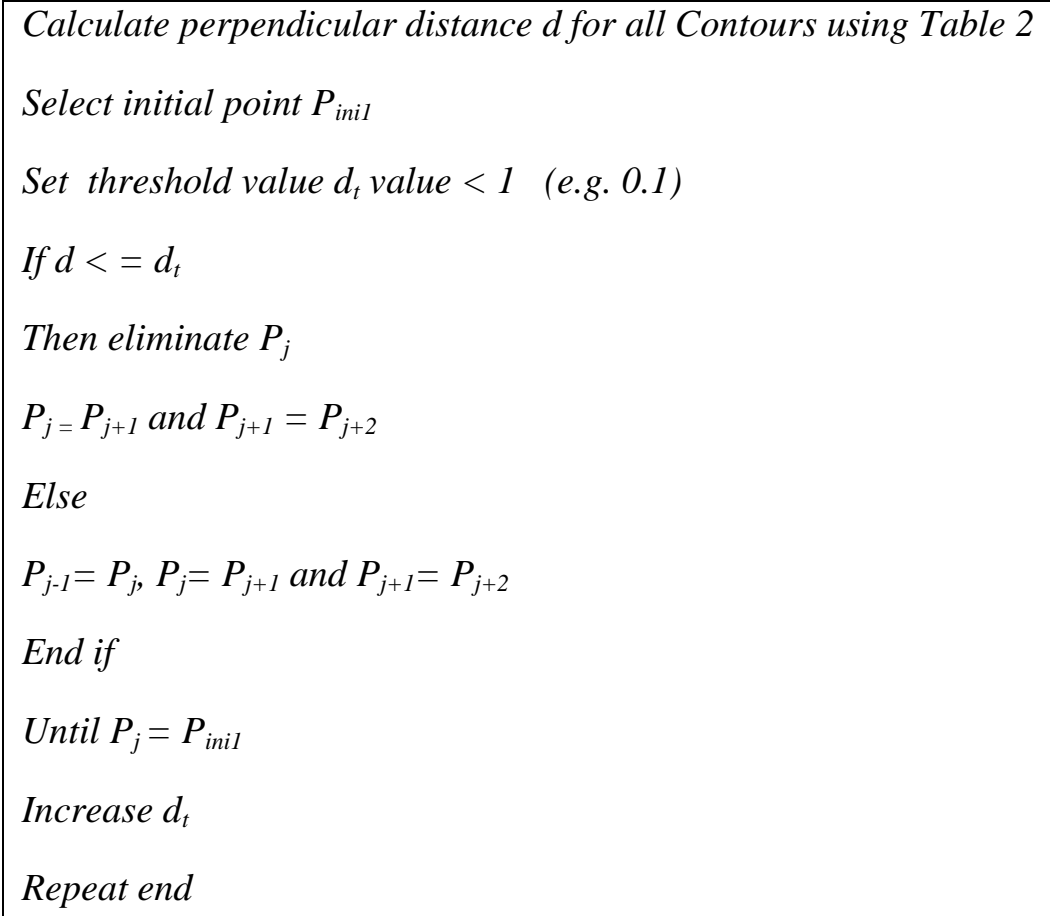


The termination condition is based on the ratio of length associated with each deleted contour point $P_{i}$ and the maximum error obtained for each contour point $P_{j}$. A simple and efficient approach for polygonal approximation is discussed. This algorithm produces a polygon approximation with any number of dominant points as per the threshold value.

\subsection{Technique 3:}

Tanvir Parvez [3] proposed a new adaptive optimization algorithm for polygonal approximation. This technique is robust to noise and effective in polygon approximation. It concentrates on the point where its curvature falls high. With those points, the optimization procedure is applied to get the best-fitting polygon approximation. This algorithm follows the points where the level of information is high. Suppression technique is used to address the noise and produce good results. The technique works well with Arabic letters and MPEG CE7 Shape-1Part B database. The algorithm first performs binarization and smoothing using Otsu (1979) and statistical average smoothing is applied (Mahmoud, 1994). With the smoothened image, the contours are extracted using the algorithm in (Pavlidis, 1982).

The selection of breakpoints is extracted using freeman's chaincode. The set of cut-points are extracted using Constrained Collinear points (CSS) and remove redundant dominant point. To detect the final set of dominant points, the initial value goes on increasing and will reduces the set of cut-points. Finally, optimization procedure obtained the least candidate sets of points of the polygonal approximation.

Table 5: Tanvir Parvez's adaptive optimization algorithm for polygonal approximation

Smooth the binary image and extract the contour.

Apply Freeman's chaincode using table 1

Calculate the left support region and right support region for refined break points

Calculate the centroid for all break points.

Sort the dominant points based on strength first, then based on distance from the centroid to select weakest point first.

For each dominant point from the sorted list, suppress the point if all three conditions are satisfied.

Set threshold value as 0.5 , if the perpendicular distance is less than threshold.

- Triangle formed by three consecutive points must be acute triangle.

- $\quad$ For each unsuppressed point $P_{l}$ other than $P_{i}, P_{j}$ and $P_{k}$, the minimum distance from $P_{l}$ to the line-segment joining $P_{i}$ and $P_{k}$ is greater than $d_{c o l}$.

- Update the strength of the other candidate dominant points and sort them.

Repeat until we obtain optimize efficiency measure elect weakest point first. 


\section{RESULTS AND DISCUSSION}

The measures taken for comparison are $\mathrm{n}_{\mathrm{d}}$, CR, ISE, WE.

- Compression ratio (CR)

$$
\mathrm{CR}=\mathrm{n} / \mathrm{n}_{\mathrm{d}}
$$

where $\mathrm{n}$ is the number of points in the contour and $\mathrm{n}_{\mathrm{d}}$ is then number of points of the polygonal approximation.

- The Integral Square Error (ISE) is defined as

$$
I S E=\sum_{i=1}^{n} e_{i}
$$

where $\mathrm{e}_{\mathrm{i}}$ is the squared distance of ith point $\mathrm{P}$ of the approximated polygon.

- Weighted sum of square error,

$$
\mathrm{WE}=\mathrm{ISE} / \mathrm{CR}
$$

The modified version of WE measure is used by Marji and Siy (2004) as

$$
\mathrm{WE}_{\mathrm{x}}=\mathrm{ISE} / \mathrm{CR}_{\mathrm{x}}
$$

where $\mathrm{x}$ is used to control the contribution of the denominator to the overall result in order to reduce the imbalance between the two terms.

The experimental results discuss the approximated polygon for the standard shapes like chromosomes,

Table 6. Comparison of Masood's, Carmona's and Tanvir's method with standard shapes like chromosomes, leaf, semicircle and infinity.

\begin{tabular}{|l|l|c|c|c|c|c|c|}
\hline Shapes & Method & $\mathrm{n}_{\mathrm{d}}$ & $\mathrm{CR}$ & ISE & WE & WE2 & WE3 \\
\hline Chromosome $(\mathrm{n}=$ & Masood-2008 & 15 & 4 & 3.88 & 0.97 & 0.243 & 0.061 \\
\hline & Carmona-Poyato-2010 & 15 & 4 & 4.27 & 1.07 & 0.267 & 0.067 \\
\hline & Tanvir-2010 & 10 & 6 & 14.34 & 2.39 & 0.398 & 0.066 \\
\hline Leaf $(\mathrm{n}=120)$ & Masood-2008 & 23 & 5.22 & 9.46 & 1.81 & 0.347 & 0.067 \\
\hline & Carmona-Poyato -2010 & 23 & 5.22 & 10.68 & 2.05 & 0.391 & 0.075 \\
\hline & Tanvir -2010 & 21 & 5.71 & 13.82 & 2.42 & 0.423 & 0.074 \\
\hline Semicircles $(\mathrm{n}=$ & Masood-2008 & 26 & 3.92 & 4.05 & 1.03 & 0.263 & 0.067 \\
\hline & Carmona-Poyato -2010 & 26 & 3.92 & 4.91 & 1.25 & 0.319 & 0.082 \\
\hline & Tanvir -2010 & 17 & 6 & 19.02 & 3.17 & 0.528 & 0.088 \\
\hline Infinity $(\mathrm{n}=45)$ & Masood-2008 & 11 & 4.09 & 2.9 & 0.71 & 0.173 & 0.042 \\
\hline & Carmona-Poyato -2010 & 10 & 4.5 & 5.29 & 1.18 & 0.261 & 0.058 \\
\hline & Tanvir -2010 & 9 & 5 & 7.35 & 1.47 & 0.294 & 0.059 \\
\hline
\end{tabular}


International Journal of Information Technology, Modeling and Computing (IJITMC) Vol.1, No.2, May 2013
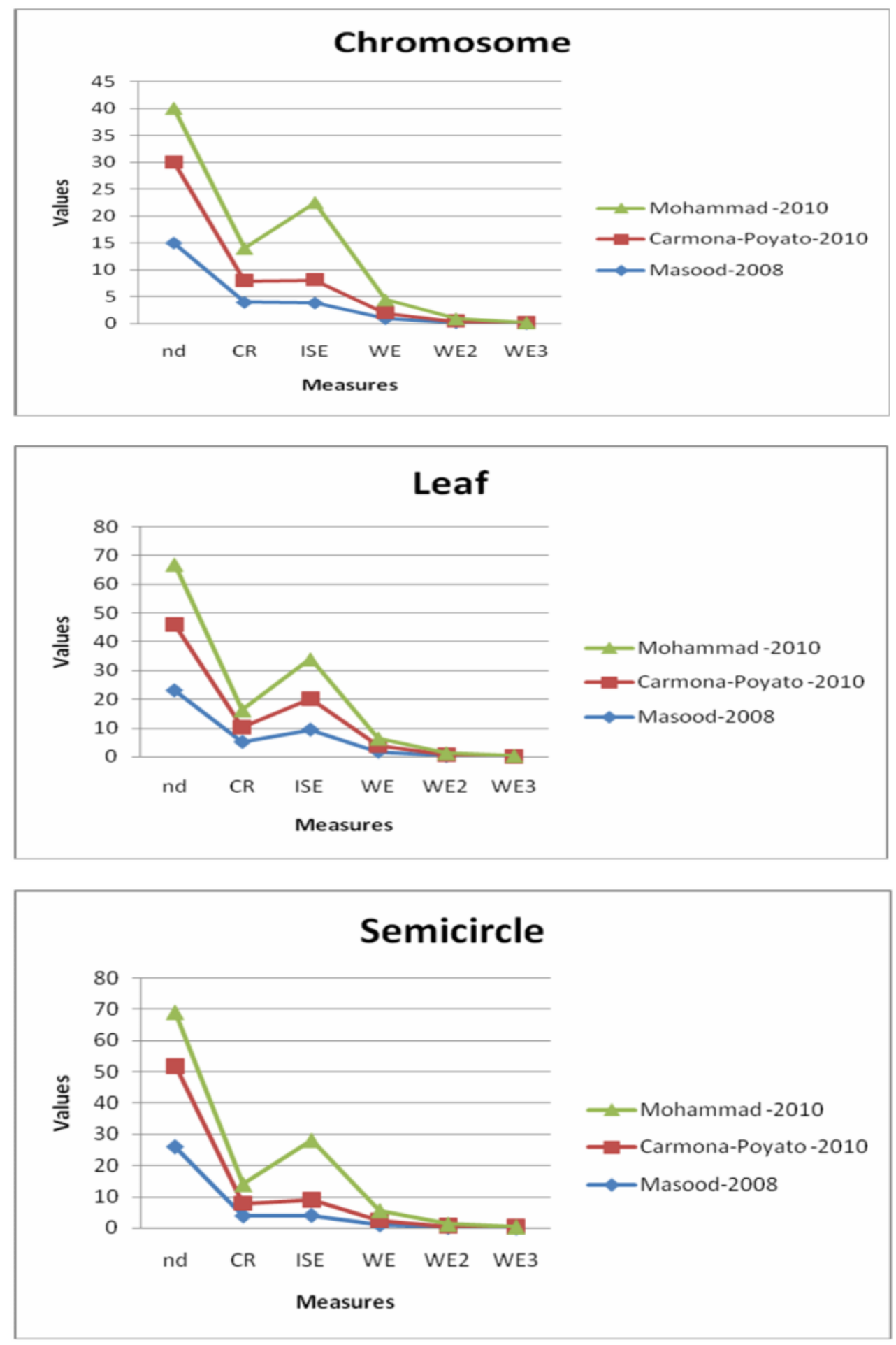


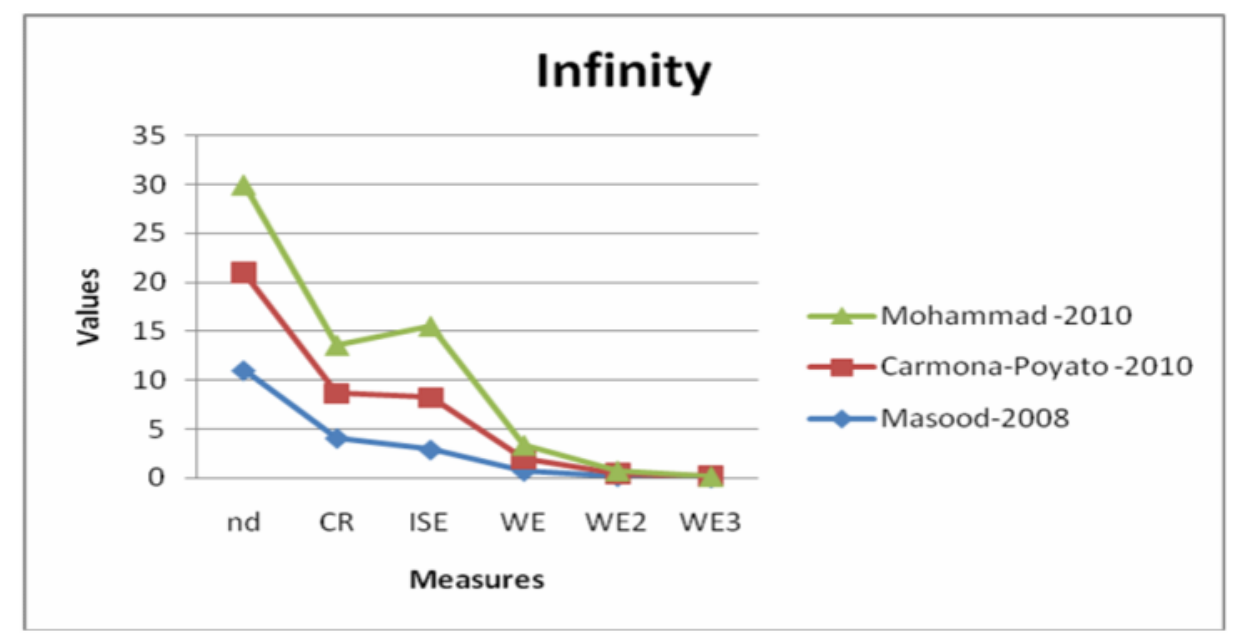

Fig 3.Graph shows the performance f three methods for the standard shape.

leaf, semicircle and infinity. For the comparison, the standard datasets like chromosomes, leaf, semicircle and infinity has been used. For the chromosome shape, Tanvir's results least number of dominant points. The ISE and WE values are high while comparing with other two techniques. For the leaf shape, Tanvir's results least number of dominant points. The measure $\mathrm{WE}_{3}$ of Masood's results is better while comparing with other two techniques. For the semicircle shape, Tanvir's results least number of dominant points. Masood's result lowest $\mathrm{WE}_{3}$ while comparing with other two techniques. For the infinity shape, Tanvir's results least number of dominant points. Masood's results least $\mathrm{WE}_{3}$ while comparing wither two techniques.

Table 7. The results comparison of three techniques with standard datasets

\begin{tabular}{|c|c|c|}
\hline a. Masood's results & b. Carmano's results & c. Tanvir's results \\
\hline & $\because$ & 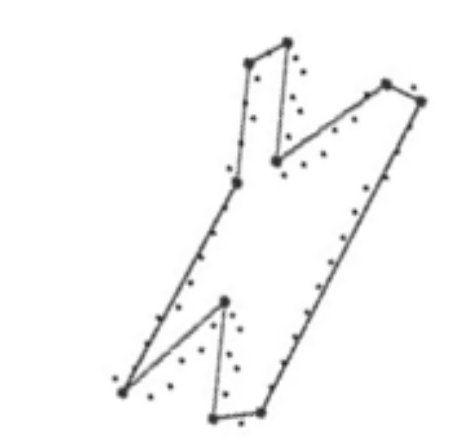 \\
\hline $\begin{array}{l}\text { a. chromosome } \\
\text { approximated dominant } \\
\text { points }\left(\mathrm{n}_{\mathrm{d}}=15\right) \text {. }\end{array}$ & $\begin{array}{l}\text { b. chromosome approximated } \\
\text { dominant points }\left(n_{d}=12\right)\end{array}$ & $\begin{array}{l}\text { c. chromosome approximated } \\
\text { dominant points }\left(\mathrm{n}_{\mathrm{d}}=10\right)\end{array}$ \\
\hline
\end{tabular}



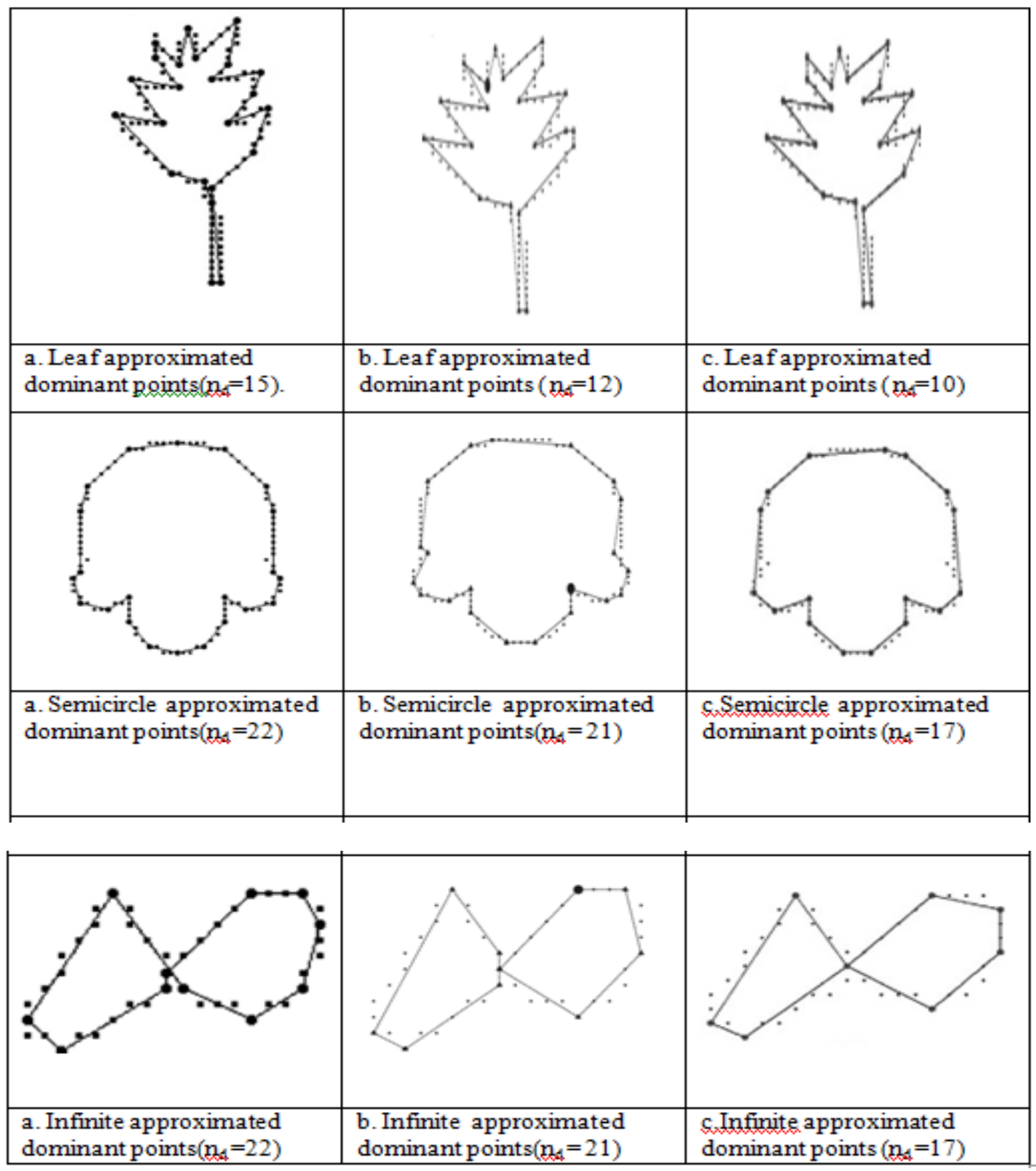

\section{CONCLUSION}

In this paper, the surveys of the three techniques are clearly illustrated and the results are compared with suitable measures. This paper fully focuses on the ideas which are explored in each technique are gathered and are elaborately explained with suitable datasets. With the help of graph the measures for each shape are calculated and are compared with other techniques. 


\section{REFERENCES}

[1] Carmona-Poyato, A., Madrid-Cuevas, F.J., Medina-Carnicer, R., Muñoz-Salinas, R.,Polygonal approximation of digital planar curves through break point suppression. Pattern Recognition 43 (2010), 14-25.

[2] A. Masood, Dominant point deletion by reverse polygonization of digital curves, Image and Vision Computing 26 (2008) 702-715.

[3] Mohammad Tanvir Parvez, Sabri A. Mahmoud, Polygonal approximation of digital planar curves through adaptive optimizations, Pattern Recognition 31 (2010)1997-2005.

[4] F. Attneave, Some informational aspects of visual perception, Psychological Review 61 (1954) 189_ 193.

[5] S. Hsin-Teng, H. Wu-Chih, A rotationally invariant two-phase scheme for corner detection, Pattern Recognition 29 (1996) 819-828.

[6] A. Melkman, J. O'Rourke, On polygonal chain approximation, in: G.T. Toussaint (Ed.), Computational Morphology, North-Holland, Amsterdam, 1998, pp. 87-95.

[7] S. Goyal, M.P. Kumar, C.V. Jawahar, P.J. Narayanam, Polygon approximation of closed curves across multiple views, in: Indian Conference on Vision, Graphics and Image Processing, 2002.

[8] P.A. Semyonov, Optimized unjoined linear approximation and its application for Eog-biosignal processing, in: 12th IEEE International Conference on Engineering.

[9] R. Neumann, G. Teisseron, Extraction of dominant points by estimation of the contour fluctuations, Pattern Recognition 35 (2002) 1447-1462.

[10] X. Hu, N. Ahuja, Matching point features with ordered geometric, rigidity, and disparity constraints, IEEE Transactions on Pattern Analysis and Machine Intelligence 16 (1994) 1041-1049

[11] I.K. Sethi, R. Jain, Finding trajectories of feature points in a monocular image sequence, IEEE Transactions on Pattern Analysis and Machine Intelligence 9(1987) 56-73.

[12] P.C. Yuen, Dominant points matching algorithm, Electronic Letters 29 (1993)2023-2024.

[13] J.C. Perez, E. Vidal, Optimum polygonal approximation of digitized curves, Pattern Recognition Letters 15 (1994) 743-750.

[14] A. Pikaz, I. Dinstein, Optimal polygonal approximation of digital curves, Pattern Recognition 28 (1995) 373-379.

[15] M. Salotti, An efficient algorithm for the optimal polygonal approximation of digitized curves, Pattern Recognition Letters 22 (2001) 215-221.

[16] M. Salotti, Optimal polygonal approximation of digitized curves using the sum of square deviations criterion, Pattern Recognition 35 (2002) 435-443 (Marc Salotti).

[17] MajedMarji, Pepe Siy, Polygonal representation of digital planar curves through dominant point detection - a nonparametric algorithm, pattern recognition 37,2004,2113-2130.

[18] Pavlidis, T., 1982. Algorithms for Graphics and Image Processing. Computer Science Press, Rockville, MD. 